\author{
EISSN:2706-7920 ISSN: 2077-4435 \\ DOI: $10.36632 / \mathrm{csi} / 2021.10 .4 .48$ \\ Journal homepage: www.curresweb.com \\ Pages: 569-574
}

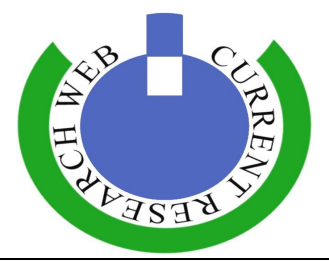

\title{
Seroprevalence of Helicobacter pylori infection among students of the Higher Institute of Technical Sciences, Msallata, Libya
}

\begin{abstract}
Abu baker H. Abduelrhman
Department of Medical Laboratory Technology, High Institute of Science and Medical Technology,
\end{abstract} EL-Garahbolii. Libya.

Received: 15 Oct. $2021 \quad$ Accepted: 05 Nov. $2021 \quad$ Published: 10 Nov. 2021

\begin{abstract}
Objective: Seroprevalence of Helicobacter pylori $(\mathrm{H}$. pylori) infection among undergraduate students at Higher Institute of Science and Technology, Msallata. Methods: A total of 150 serum samples were collected from students of the Higher Institute of Science and Technology in Msallata, with 100 $\mathrm{L}$ of each sterile serum transferred to a sample of $H$. pylori antigens kit $(H$. pylori Antigen KitClinotech, USA). After 10 minutes, two distinct red lines in the group's control and test regions indicate a positive reaction. The isolated H.pylori from clinical blood was identified according to their morphological, cultural characteristics and consumption of broth manual some biochemical tests and confirmed by VITIK2 system. Results: A total of 63 (42.66\%) of the 150 students tested positive for the virus. The prevalence of $H$. pylori infection was found to be related to age in a study of $H$. pylori seroprevalence. Infection rates were 45.5 percent among students aged 18-20, 85.7 percent among adults aged 31-40, 66.7 percent among those aged 41-50, and 28.6 percent among those aged 51 and up. Gender, age, and type of infection (symptomatic or asymptomatic seropositive infection) $(P<0.05)$ all showed statistically significant differences (using Chi-square). The Biochemical characteristics of H. pylori was confirmed with excellent probability $99 \%$ after full Biochemical identification by VITIK2 system as well as the susceptibility information. Conclusions: This microorganism should be recognized as a possible cause of illness in children by community health personnel. Furthermore, the mode of transmission and possible methods of controlling the bacterial infection among students or in a community are public health concerns that need to be investigated further.
\end{abstract}

Keywords: Helicobacter pylori, infection, students, Libya

\section{Introduction}

Helicobacter pylori (H. pylori) is a major microorganism responsible for the development of a wide range of gastroduodenal diseases (Parsonnet et al., 1991).

H. pylori is a gram-negative, microaerophilic bacterium with a helix that causes chronic lowlevel inflammation of the stomach lining and has been linked to the development of duodenal and gastric ulcers as well as stomach cancer. More than half of the world's population has H. pylori in their upper gastrointestinal tract, with more than $80 \%$ of those having asymptomatic infection. It is now the world's most common infection (Parsonnet et al., 1991).

Infection with $H$. pylori is most commonly acquired during childhood and can last a lifetime if not treated (Torres et al., 2000). According to seroepidemiologic studies, serum antibodies against $H$. pylori are present in $50 \%$ of adults in developed countries and nearly $90 \%$ of adults in developing countries (Chi et al., 2009). Despite its high prevalence, little is known about the exact route of infection transmission in our society (Queiroz and Luzza, 2006).

As a result, knowing the prevalence of this infection is important. The objective of this research is to find out the prevalence of $H$. pylori infection among undergraduate students in Msalata Municipality, Libya.

Corresponding Author: Abu baker H. Abduelrhman, 1Department of Medical Laboratory Technology, high institute of science and Medical technology, EL-Garahbolii. Libya.

E-mail: hmeda63@gmail.com 


\section{Materials and Methods}

This study was conducted during the period from 2020-2021 at Bacteriology, labs, Department of Medical Laboratory Technology, high institute of science and Medical technology, Msalata, Libya.

\section{Collection clinical specimens}

Blood specimens were collected from 150 Higher diploma students, gender male and female and age (table, 1) at the Higher Institute of Science and Technology in Msallata Municipality from 8:00 a.m. to 12:00 p.m. in the laboratories section. They were asked to complete surveys in order to provide basic information such as their gender (male and female) and age (year).

Table 1: Risk factor of $H$. pylori infection higher diploma students regarding sex and age group.

\begin{tabular}{lccc}
\hline Rick factor & & Number of Higher diploma students (No= 150) \\
\hline \multirow{2}{*}{ Gender } & Male & $70 / 150$ & $46.66 \%$ \\
& Female & $80 / 150$ & $53.33 \%$ \\
\hline \multirow{2}{*}{ Age (year) } & $\mathbf{1 8 - 2 0}$ & $66 / 150$ & $44.00 \%$ \\
& $\mathbf{2 1 - 2 3}$ & $84 / 150$ & $56.00 \%$ \\
\hline
\end{tabular}

\section{Precaution clinical Blood}

Safety precautions: Gloves and lab coat were wearied before beginning work. All biologic agents and chemicals were disposed in accordance with local environmental and safety regulations. These procedures can be found in the following WHO (2004).

\section{Collecting clinical specimens}

The Higher diploma students were selected randomly from blood donors. Ten $\mathrm{ml}$ of venous blood samples were obtained by peripheral vein puncture under aseptic precaution from all students. Two $\mathrm{ml}$ of blood on sodium citrate to perform pro thrombin time. The blood specimens were refrigerated and arrived to the laboratory through one hour. The rest of blood was drawn in plain tube then put in water path at $37 \mathrm{C}^{\circ}$ for 30 minutes then centrifuged at $3000 \mathrm{rpm}$ for 10 minutes then the resultant serum was divided in aliquots and stored at $-20 \mathrm{C}^{\circ}$ for analysis.

\section{Serological assay}

Using a sterile dispensable dropper, 3 drops of serum (approximately $100 \mathrm{~mL}$ ) were transferred to the wells of the test kit each time (H. pylori Antigen Kit-Clinotech, USA). All sera that were not used were stored in a refrigerator. After 10 minutes, the test was conducted. The occurrence of two distinct red lines on the kit's control and test regions represented.

\section{Isolation and enumeration of $\boldsymbol{H}$. pylori}

Aseptically $(5 \mathrm{~g})$ of clinical blood sample was mixed with $95(\mathrm{ml})$ of sterile buffer peptone water and incubated in $\mathrm{CO}_{2}$ incubator at $35^{\circ} \mathrm{C}$ for $24 \mathrm{~h}$ (Al-Sulami et al., 2008). One to ten (ml) mixture was transferred to nutrient broth and incubated in $\mathrm{CO}_{2}$ incubator at $35^{\circ} \mathrm{C}$ for $72 \mathrm{~h}$. The enumerated cultures were re inoculated on MRS broth (FDA, 2002) selective medium and incubated in $\mathrm{CO}_{2}$ incubator at $37^{\circ} \mathrm{C}$ for $24 \mathrm{~h}$. then put in refrigerator for further identification.

\section{Purification and identification of isolated $\mathrm{H}$. pylori.}

Bacterial colonies obtained from all previously mentioned media were chosen and picked up according to variation in culture characteristics and colony formation then purified by streak-plate method on Nutrient agar medium. Pure isolates were maintained on slants of the MRS broth medium at $4^{\circ} \mathrm{C}$ for subsequent identification. Almost all microscopically examinations (Morphological characters: Shape, texture) and biochemical testing (Biochemical tests (Gram reaction, Motility test, Catalase test and Oxidase test) were carried out according to Bergey's manual, (2009); Collins \& Lyne (2004) and Cheesbrough (2006). 


\section{Haemolysis on blood agar medium}

Blood haemolysis ability of the isolated bacterial colonies was tested using tryptic soy agar obtained from (Difco, USA), supplemented with 5\% sterile human blood. Bacterial growth on blood agar medium showed the following features: a-haemolysis, $\beta$-haemolysis and no haemolysis.

Isolated $H$. pylori identification was confirmed by VITEK2 system carried out according to Shetty \& Turner (1998) and Funke et al. (1998). The reagent cards have 64 wells that can each contain an individual test substrate. Substrates measure various metabolic activities such as acidification, alkalinization, enzyme hydrolysis, and growth in the presence of inhibitory substance. There are currently four reagent cards available for the identification of different organism as follows:

\section{Culture Requirements}

The parameters include acceptable culture media, culture age, incubation conditions and inoculums turbidity.

\section{Suspension preparation}

A disposable bacterial needle used to transfer a single colony of a pure H.pylori culture and to suspended the H.pylori in $3.0 \mathrm{ml}$ of sterile saline (aqueous $0.45 \%$ to $0.50 \% \mathrm{NaCl}, \mathrm{pH} 4.5$ to 7.0 ) in a $12 \times 75 \mathrm{~mm}$ clear plastic (polystyrene) test tube. The turbidity was adjusted according Table (2) and measured using a turbidity meter called the DensiChek.

Table 2: Suspension turbidities used for card inoculation

\begin{tabular}{cc}
\hline Product & McFarland Turbidity Range \\
\hline GN & $0.50-0.63$ \\
GP & $0.50-0.63$ \\
YST & $1.80-2.20$ \\
BCL & $1.80-2.20$ \\
\hline
\end{tabular}

\section{Inoculation}

Identification cards were inoculated with isolated $H$. pylori suspensions using an integrated vacuum apparatus. A test tube contain in $g$ the microorganism suspension was placed into especial rack (cassette) and the identification card was placed in the neighbor in gloat while inserting the transfer tube into the corresponding suspension tube.

\section{Antibiotic sensitivity test: (Vitek 2 system was used to antibiotic sensitivity test}

Cefaclor, Cefotaxime, Cefoperazone, Cefepime, Clindamycin, Imipenem, Doxycycline, Levofloxacin, Ciprofloxacin, Amikacin, Sulphamethoxazole/Trimesoprim, Azithromycin, Ampicillin, Amoxicillin/Clavulinic acid, Pipracillin/Tazobactam and Nitrofurantion).

\subsection{Statistical analysis}

The Chi-squares (2) test was used $\mathrm{P}<0.05$ was considered significant.

\section{Result}

\subsection{H. pylori detection}

H. pylori seropositivity was found in 63 of the 150 undergraduate students by Cast gram Rapid chromatographic Immunoassay (H. pylori Antigen Kit-Clinotech, USA). The positive samples were distinct pink colored band appeared on test regions, in addition to a pink line on the control line region, with a seroprevalence rate of $42.66 \%$. Males were infected at a rate of $58.57 \%(36 / 70)$, while females were infected at a rate of $33.75 \%(27 / 80)$. Males and females had significantly different levels of H. pylori infection (P 0.0286).

It was discovered that the prevalence of $\mathrm{H}$. pylori infection among students was age-related. Students in grades $18-20$ had the lowest $\mathrm{H}$. pylori rate $(23 / 63,36.50 \%)$, while students in grades 21 23 had the highest no significant rate $(40 / 63,49.63 \%) 0.1175$. 
The males accounted for $42.9 \%$ of the 108 seropositive students, while the females accounted for $23.1 \%$. Females accounted for $76.9 \%$ of the 72 asymptomatic students studied, while males accounted for $57.1 \%, 0.189$.

Table 3: Risk factor of $\mathrm{H}$. pylori infection among higher diploma students regarding sex and age group.

\begin{tabular}{cccc}
\hline Parameter & Positive $(\mathbf{n}=\mathbf{6 3})$ & Negative $(\mathbf{n}=\mathbf{8 7})$ & $\mathbf{P}<\mathbf{0 . 0 5}$ \\
\hline Sex & & & \\
Male & $36(58.57 \%)$ & $34(39.08 \%)$ & 0.0286 \\
Female & $27(33.75 \%)$ & $53(60.91 \%)$ & \\
Asymptomatic & & & 0.189 \\
Male & $9 / 36(30.55 \%)$ & 34 & \\
Female & $13 / 27(48.14 \%)$ & 53 & 0.1175 \\
Age (yr) & $23(36-50 \%)$ & $43(49.42 \%)$ & \\
$\mathbf{1 8 - 2 0}$ & $40(63.49 \%)$ & $44(50.57 \%)$ & \\
$\mathbf{2 1 - 2 3}$ & &
\end{tabular}

\subsection{Isolated $H$. pylori identification}

In the present work, the isolated $H$. pylori from clinical blood was identified according to their morphological, cultural characteristics and consumption of broth manual some biochemical tests according to Bergey's manual, (2009) and confirmed by VITIK2 system. The morphological characters of $H$. pylori were straight rods to long spiral shape, motility, colonies were translucent to pale grayish and smooth texture. Biochemical characteristics were Gram negative, Beta hemolysis on blood agar, Facilitative anaerobic, positive, coagulase, catalase, oxidase and urease. The Biochemical characteristics of $H$. pylori was confirmed with excellent probability $98 \%$ after full Biochemical identification by VITIK2 system as well as the susceptibility information was provided as shown in table ( 4 ).

Microbiology Chart Report

BioMérieux Customer: 1005818356

Organism Quantity: $1000000 \mathrm{cfu} / \mathrm{mL}$

Selected Organism: Helicobacter pylori

Table 4: Biochemical characteristics of H. pylori by VITEK 2

\begin{tabular}{lcc}
\hline Identification Information & Analysis Time: 5.80 hours & Status: Final \\
Selected Organism & $99 \%$ Probability & Helicobacter pylor \\
& Bionumber: & 0003453103500250
\end{tabular}

\begin{tabular}{llllllllllll}
\hline \multicolumn{10}{c}{ Data of Biochemical tests for Helicobacter pylori } \\
APPA & - & ADO & - & PyrA & - & ARL & - & dCEL & - & BGAL & - \\
H2S & - & BNAG & - & AGLTp & - & dGLU & + & GGT & + & OFF & - \\
BGLU & - & dMAL & - & dMAN & + & dMNE & + & BXYL & - & BAlap & + \\
ProA & + & LIP & + & PLE & - & TyrA & + & URE & - & dSOR & - \\
SAC & - & dTAG & - & dTRE & - & CIT & + & MNT & + & $5 K G$ & - \\
ILATk & + & AGLU & - & SUCT & + & NAGA & - & AGAL & - & PHOS & - \\
GlyA & - & ODC & - & LDC & - & lHISa & - & CMT & + & BGUR & - \\
O129R & + & GGAA & - & lMLTa & + & ELLM & - & lLATa & - & & \\
\hline
\end{tabular}

Installed VITEK 2 ® Systems Version: 08.01

\subsection{Antibiotic sensitivity of Helicobacter pylori isolate}

According to VITEK 2, the antimicrobials sensitivity of $H$. pylori showed a range of susceptibilities ranging from sensitive to intermediate to resistant to the various antibiotics tested shown in table (5). 
Table 5: Antibiotic sensitivity of Helicobacter pylori by VITEK 2.

\begin{tabular}{|c|c|c|c|c|c|}
\hline \multicolumn{6}{|c|}{ Data of Antibiotic sensitivity for Helicobacter pylori and MIC } \\
\hline Antimicrobial & MIC & Interpretation & Antimicrobial & MIC & Interpretation \\
\hline Ampicillin & $>=32$ & $\mathrm{R}$ & Imipenem & 0.5 & $\mathrm{~S}$ \\
\hline Amoxicillin / Clavulanic Acid & $>=32$ & $\mathrm{R}$ & Amikacin & $>=64$ & $\mathrm{R}$ \\
\hline Ticarcillin & $>=128$ & $\mathrm{R}$ & Gentamicin & $>=16$ & $\mathrm{R}$ \\
\hline Piperacillin/Tazobactam & 64 & I & Tobramycin & $>=16$ & $\mathrm{R}$ \\
\hline Cefalotin & $>=64$ & $\mathrm{R}$ & Nalidixic Acid & $>=32$ & $\mathrm{R}$ \\
\hline Cefoxitin & $>=64$ & $\mathrm{R}$ & Ciprofloxacin & $>=4$ & $\mathrm{R}$ \\
\hline Cefotaxime & $>=64$ & $\mathrm{R}$ & Ofloxacin & $>=8$ & $\mathrm{R}$ \\
\hline Ceftazidime & $>=64$ & $\mathrm{R}$ & Nitrofurantoin & 256 & $\mathrm{R}$ \\
\hline Ertapenem & & & Trimethoprim/ & & \\
\hline & $>=8$ & $\mathrm{R}$ & $\begin{array}{c}\text { Sulfamethoxazo } \\
\text { le }\end{array}$ & $>=320$ & $\mathrm{R}$ \\
\hline
\end{tabular}

Installed VITEK 2 ® Systems Version: 08.01

MIC Interpretation Guideline: Global CLSI-based

Therapeutic Interpretation Guideline: NATURAL RESISTANCE

AES Parameter Set Name: Global CLSI-based+

Natural Resistance AES Parameter Last Modified: Oct 24, 2017 14:16 CDT

\section{Discussion}

H. pylori is one of the most common bacterial infections in humans, and it affects children in developing countries at a young age. Endoscopy and serology are two traditional diagnostic tools that can be too invasive for children, but the (Robert et al., 1993).

The results of this study showed that the seroprevalence of $H$. pylori was $42.66 \%$ among undergraduate students at the Higher Institute of Science and Technology Msallata, which is consistent with many studies in some developing countries (Jafri et al., 2010). This seroprevalence rate, however, was lower than that reported in (Chi et al., 2009; Ishaleku and Hope 2010).

This pathogenic bacteria infection is acquired during childhood, and its prevalence rises with age, with the patient's infection lasting for the majority, if not all, of his or her life (Jafri et al., 2010). The fact that males have more symptomatic $H$. pylori infections than females is usually related to other risk factors such as stress and smoking, which aggravate $H$. pylori infections, according to (Zhang et al., 2010).

Females have a higher prevalence of asymptomatic $H$. pylori infection than males, possibly because females engage in more social activities than males, according to (Queiroz and Luzza, 2006).

As a result, community health workers should be aware of this microbe as a possible source of illness in children. They can help by promoting good hygiene and educating families about how to prevent children from contracting the bacteria. Furthermore, the mode of transmission and potential means of controlling the bacterial infection among students or a community are public health concerns that need to be investigated further.

Our results demonstrated the antimicrobial susceptibility of $H$. pylori with a range of sensitivities ranging from sensitive to moderate to resistance to different antibiotics tested according to several similar studies (Wheeldon et al., 2004) and (Ziemniak, 2006).

\section{References}

Al-Sulami, A. et al., 2008. Primary isolation and detection of Helicobacter pylori from dyspeptic patients: a simple, rapid method. Eastern Mediterranean Health Journal, 14 (2):268-276.

Bergey's Manual of Systematic Bacteriology, 2009. Is it an invaluable reference work for microbiologists?" The Second National Conference SIMTREA., 17.

Cheesbrough, M., 2006. District Laboratory Practice in Tropical Countries (2 ${ }^{\text {nd }}$ Edition). London English Language Book Society, 100-194.

Chi, H., M.J. Bair, M.S. Wu, N.C. Chiu, Y.C. Hsiao, and K. Chang, 2009. Prevalence of Helicobacter pylori infection in high-school students on Lanyu Island, Taiwan: risk factor analysis and effect on growth. J. Formos. Med. Assoc., 108(12): 929-36. 
Collins, C.H. and P.M. Lyne, 2004. Microbiological Methods. Milk, Dairy Produce, Eggs and IceCream. Eight Edition, Arnold Publishers, London, 234-244.

FAO/WHO, 2002. Food and Agriculture Organization, World Health Organization. Guidelines for the evaluation of probiotics in food: report of a joint FAO/WHO working group on drafting guidelines for the evaluation of probiotics in food. London.

Funke, G., P.A. Lawson, F.S. Nolte, N. Weiss, and M.D. Collins, 1998. Aureobacterium resistens sp. nov., exhibiting vancomycin resistance and teicoplanin susceptibility. FEMS Micobiol Lett., 158: 89-93.

Ishaleku, D., and A.I. Hope, 2010. "Seroprevalence of Helicobacter pylori infection among students of a Nigerian University." Asian Pacific Journal of Tropical Medicine 3.7: 584-585.

Jafri, W., J. Yakoob, S. Abid, S. Siddiqui, S. Awan, S.Q. Nizami, 2010. Helicobacter pylori infection in children: population-based agespecific prevalence and risk factors in a developing country. Acta Paediatr., 99(2): 279-82.

Parsonnet, J., G.D. Friedman, D.P. Vandersteen, et al., 1991. Helicobacter pylori infection and the risk of gastric carcinoma. N. Engl. J. Med., 325:1127-31.

Queiroz, D.M.M., and F. Luzza, 2006. Epidemiology of Helicobacter pylori infection. Helicobacter. 2006 Oct;11 Suppl 1:1-5. doi: 10.1111/j.1478-405X.2006.00429.x.

Robert, J.H., A.V. Pablo, F. Catterine, O. Jimena, P. Priscilla, S. Viviana, G.R. Robert, S.W. Steven, J.Jr. Glenn Morris, 1993. Seroprevalence of Helicobacter pylori in Chile: Vegetables May Serve as One Route of Transmission. The Journal of Infectious Diseases, 168(1): 222-226.

Shetty, A.K., and D.A. Turner, 1998. In vitro survival and differentiation of neurons derived from epidermal growth factor-responsive postnatal hippocampal stem cells: Inducing effects of brain-derived neurotrophic factor. J. Neurobiol., 35:395-425.

Torres, J., G. Perez-Perez, K.J. Goodman, et al., 2000. A comprehensive review of the natural history of Helicobacter pylori infection in children. Arch. Med. Res., 31:431-69.

Wheeldon, T.U., M. Granström, T.T. Hoang, D.C. Phuncarg, L.E. Nilsson, and M. Sörberg, 2004. The importance of the level of metronidazole resistance for the success of Helicobacter pylori eradication. Aliment Pharmacol Ther. Jun 15; 19(12):1315-21. doi: 10.1111/j.13652036.2004.01959.x. PMID: 15191514.

WHO, 2004 World Health Organization. Laboratory Biosafety Manual, Third edition. http://www.who.int/csr/delibepidemics/WHO_CDS_CSR_LYO_2004_11/en/.

Zhang, L., G.D. Eslick, H.H. Xia, C. Wu, N. Phung, and N.J. Talley, 2010. Relationship between alcohol consumption and active Helicobacter pylori infection. Alcohol Alcohol, 45(1): 89-94.

Ziemniak, W., 2006. Efficacy of Helicobacter pylori eradication taking into account its resistance to antibiotics. J. Physiol Pharmacol. Sep., 57 Suppl 3:123-41. PMID: 17033111. 